\title{
Quantum Phase Diagram of One-Dimensional Spin and Hubbard Models with Transitions to Bond Order Wave Phases ${ }^{\dagger}$
}

\author{
Manoranjan Kumar, ${ }^{\text {a }}$ S. Ramasesha, ${ }^{\mathrm{b}}$ and Zoltán G. Soos ${ }^{\mathrm{c}, *}$ \\ ${ }^{a}$ S. N. Bose National Centre for Basic Sciences, Block-JD, Sector-III, Salt Lake, Kolkata-700098, India \\ ${ }^{\mathrm{b}}$ Solid State and Structural Chemistry Unit, Indian Institute of Science, Bangalore 560012, India \\ 'Department of Chemistry, Princeton University, Princeton NJ 08544, USA \\ RECEIVED JULY 3, 2013; REVISED AUGUST 30, 2013; ACCEPTED SEPTEMBER 2, 2013
}

\begin{abstract}
Similar quantum phase diagrams and transitions are found for three classes of one-dimensional models with equally spaced sites, singlet ground states (GS), inversion symmetry at sites and a bond order wave (BOW) phase in some sectors. The models are frustrated spin $-1 / 2$ chains with variable range exchange, half-filled Hubbard models with spin-independent interactions and modified Hubbard models with site energies for describing organic charge transfer salts. In some range of parameters, the models have a first order quantum transition at which the GS expectation value of the sublattice spin $\left\langle S_{\mathrm{A}}{ }^{2}\right\rangle$ of odd or even-numbered sites is discontinuous. There is an intermediate BOW phase for other model parameters that lead to two continuous quantum transitions with continuous $\left\langle S_{\mathrm{A}}{ }^{2}\right\rangle$. Exact diagonalization of finite systems and symmetry arguments provide a unified picture of familiar 1D models that have appeared separately in widely different contexts. (doi: $10.5562 /$ cca2324)
\end{abstract}

Keywords: bond order wave phases, quantum phase transitions, frustrated spin chains, extended Hubbard models, neutral-ionic transition models

\section{INTRODUCTION}

Phase transitions occur in the thermodynamic limit of interacting many-body systems. Thermodynamic phase transitions reflect competition between internal energy and entropy that depends on parameters such as temperature, pressure or volume. The Gibbs free energy $G(T, P)$ or Helmholtz free energy $A(T, V)$ are state functions that describe phase transitions of one-component systems. By definition, quantum phase transitions occur at absolute zero in the ground state (GS) of infinite systems whose Hamiltonian has at least two non-commuting operators. The GS energy per particle, $E_{0}(X, Y)$, depends on model parameters $X, Y$. In complete analogy with thermodynamic free energies, $E_{0}(X, Y)$ is a continuous function that is doubly degenerate at the boundary $X_{\mathrm{c}}, Y_{\mathrm{c}}$ of two quantum phases. The transition is first order or continuous, respectively, when first derivatives of $E_{0}(X, Y)$ with respect to $X$ or $Y$ are discontinuous or continuous at $X_{\mathrm{c}}, Y_{\mathrm{c}}$. The major challenge is to obtain the exact GS of infinite quantum systems. Exact $E_{0}(X, Y)$ are largely limited to one-dimensional (1D) models, and even then derivatives of $E_{0}(X, Y)$ are rarely known.
In principle, $E_{0}(X, Y)$ per particle yields quantum phase diagrams for models with three parameters, one of which sets the energy scale. In this paper we discuss the quantum phase diagrams of three classes of 1D models, all with equally spaced sites, including prototypical special cases that have been extensively studied separately. We rely on exact diagonalization (ED) of finite models and mention other methods as needed. We point out some striking similarities among phase diagrams of models that support a bond order wave (BOW) phase in some parameter range of $X$ and $Y$. The BOW phase has broken inversion symmetry and finite energy gap $E_{\mathrm{m}}$ from the singlet GS to the lowest triplet state. 1D models are paramagnetic with finite magnetic susceptibility at absolute zero when $E_{\mathrm{m}}=0$ or diamagnetic with vanishing magnetic susceptibility when $E_{\mathrm{m}}>0$.

The first class is $E_{0}(U, V)$ in half-filled Hubbard models with on-site repulsion $U>0$, transfer integral $t=1$ and spin independent interactions $V$ of arbitrary range. Not so long ago, Nakamura ${ }^{1}$ realized that the extended Hubbard model (EHM) with $V>0$ between neighbors has a narrow BOW phase that has since been confirmed by multiple methods, ${ }^{2-5}$ although the $U, V$ phase diagram is still approximate. $E_{0}(U, V)$ is doubly

\footnotetext{
$\dagger$ Dedicated to Professor Douglas Jay Klein on the occasion of his $70^{\text {th }}$ birthday.

* Author to whom correspondence should be addressed. (E-mail: soos@Princeton.EDU)
} 
(a)

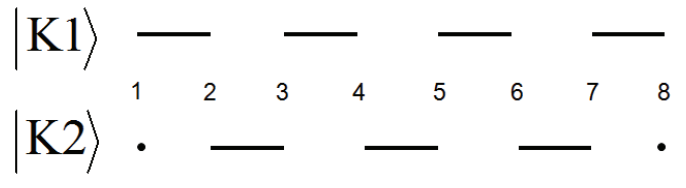

(b)

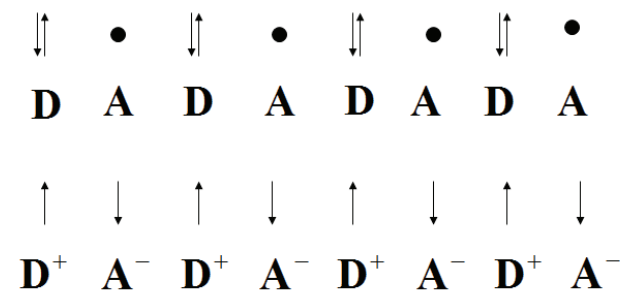

Figure 1. (a) Schematic representation of adjacent singlet paired spins, $(\alpha \beta-\beta \alpha) \cdot 2^{-0.5}$, in the two Kekule structures $|\mathrm{K} 1\rangle$ and $|\mathrm{K} 2\rangle$ of an infinite chain of $s=1 / 2$ spins; the sites are equally spaced; sites 1 and $N=4 n$ are singlet paired in $|\mathrm{K} 2\rangle$. (b) Schematic representation of a neutral donor-acceptor chain with two electrons in the HOMO of $\mathbf{D}$ and a radical ion chain with one electron transferred to the LUMO of $\mathbf{A}$.

degenerate in the BOW phase, inversion symmetry is broken, and $E_{\mathrm{m}}(U, V)$ to the lowest triplet state is finite. ${ }^{1}$ The second class is $E_{0}(g, 1 / \alpha)$ in spin chains ${ }^{6}$ with frustrating exchange $g$ between second neighbors and normalized nonfrustrating exchanges with range $1 / \alpha$. The $J_{1} J_{2}$ model is the short-range limit, $1 / \alpha=0$, of nearest neighbor exchange $J_{1}=1$ and $g=J_{2} / J_{1}$. Okamoto and Nomura $^{7}$ found the onset of the BOW phase at $g=$ 0.2411. Majumdar and Ghosh ${ }^{8}$ showed that the exact GS at $g_{\mathrm{MG}}=1 / 2$ are the Kekulé structures $|\mathrm{K} 1\rangle$ and $|\mathrm{K} 2\rangle$ of organic chemistry. As sketched in Figure 1a, $|\mathrm{K} 1\rangle$ and $|\mathrm{K} 2\rangle$ are two ways to form singlet pairs of adjacent $s=$ $1 / 2$ sites and clearly break inversion symmetry at sites. The third class is $E_{0}(\Gamma, V)$ in the restricted basis ${ }^{9}$ of modified Hubbard models with site energy $\pm \Gamma$, transfer integral $\mathrm{t}=1$ and electrostatic (Madelung) energy $E_{\mathrm{M}}=$ $\alpha_{\mathrm{M}} V$ for Coulomb interaction $V$ between neighbors. The model has been applied to neutral-ionic transitions ${ }^{10}$ (NIT) in organic charge transfer (CT) salts ${ }^{11-13}$ that contain mixed 1D stacks of planar $\pi$-electron donors (D) and acceptors (A). The neutral and ionic limits sketched in Figure $1 \mathrm{~b}$ have two electrons in the HOMO of $\mathbf{D}$ and one electron transferred to the LUMO of $\mathbf{A}$, respectively. Virtual transfers lift the $2^{N}$-fold spin degeneracy of the ionic state and generate a singlet GS.

Although different in many ways, these models share features that are central to their quantum phase diagrams. In addition to equally spaced sites, the total spin $S$ is conserved and GS is a singlet, $S=0$, over the entire parameter range of interest. The models have inversion symmetry $\sigma$ at sites. The excitation $E_{\sigma}$ is to the lowest-energy singlet with opposite $\sigma$ to the GS while $E_{\mathrm{m}}$ is to the lowest triplet state. The models support first order or continuous quantum phase transitions.
They are all candidates for a BOW phase where $E_{\sigma}=0$ and have fundamentally different GS in different parameter sectors. Competition among $U, V$ and $t$ in half-filled Hubbard models with both charge and spin degrees of freedom is analogous to frustration in spin chains in the sense that comparable $E_{0}(X, Y)$ is achieved with completely different properties.

The limit of no overlap between sites offers both insight and simplicity. The formation energy of ionic crystals can be viewed as a first order quantum phase transition when the Madelung energy $E_{\mathrm{M}}$ exceeds the energy $I_{\mathbf{D}}-A_{\mathbf{A}}$ to ionize the donor and transfer the electron to the acceptor. Finite overlap, and hence finite electron transfer $t$, complicates the analysis of NITs that typically involve partial electron transfer because the neutral phase is slightly polar and the ionic phase is not fully ionic. NITs may be considered to be quantum transitions since they occur at temperature that is low compared to any electronic excitation. It has long been recognized that the magnetic properties of a half-filled 1D Hubbard model with $U>4 t$, the bandwidth of a 1D tight binding or Hückel chain, are given by a linear Heisenberg antiferromagnet (HAF) with $s=1 / 2$ sites and isotropic exchange $J_{1}=4 t^{2} / U$ between first neighbors. The HAF also describes the magnetic properties of modified Hubbard models on ionic side for $t<<E_{\mathrm{CT}}$, the optical CT excitation polarized along the stack.

Klein and Seitz ${ }^{14}$ performed degenerate perturbation theory in $t / U$ on the half-filled Hubbard model. In fourth order, they obtained $J_{2}=4 t^{4} / U^{3}$ between second neighbors and reduced $J_{1}$ by $-4 t^{4} / U^{3}$. They found what would later be called spin frustration since antiferromagnetic $J_{2}>0$ is frustrating for either positive or negative $J_{1}$. The sixth order correction ${ }^{14}$ has $t^{6} / U^{5}$ contributions to $J_{1}$ and $J_{2}$ as well as an additional interaction that involves four spins. Later, van Dongen ${ }^{15}$ studied the EHM and obtained $J_{2}=4 t^{4}(1+V / U) /(U-V)^{3}$. Hirsch ${ }^{16}$ used quantum Monte Carlo to characterize the first order transition of the EHM from a Heisenberg spin chain at $V<U / 2$ to a charge density wave (CDW) with two electrons on every other site. The phase boundary is accurately given by perturbation theory ${ }^{15}$ for $U>10 t$. A motivated search using other methods such as field theory, symmetry and renormalization is needed to find the BOW phase. ${ }^{1-5}$ Frustrating $J_{2}$ is intrinsic to quantum cell models with spin-independent interactions but it is not a free parameter and both spin and charge degrees of freedom must be considered in general.

As noted above, we rely on ED of finite systems. Exact eigenstates in addition to energies compensate to a considerable extent for the limitations of finite size. We choose models with $N=4 n$ sites, equal spacing between sites, and periodic boundary conditions (PBC), thereby explicitly retaining inversion symmetry $\sigma$ at sites and ensuring integer $S \leq 2 n$. There are several 


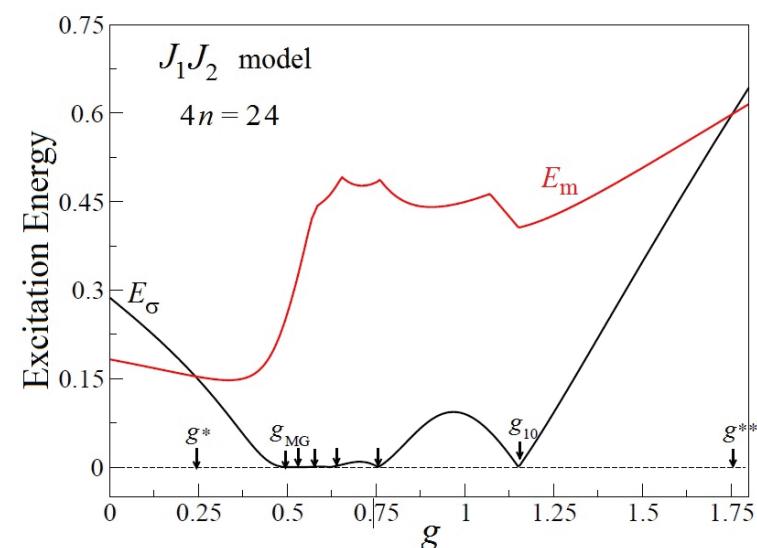

Figure 2. Excitation energies $E_{\mathrm{m}}$ and $E_{\sigma}$ with increasing frustration $g$ in the 24-spin $J_{1} J_{2}$ model, Eq. $1 ; E_{\mathrm{m}}$ is to the lowest triplet, $E_{\sigma}$ is to the lowest singlet with reversed inversion symmetry. Arrows indicate $\mathrm{g}$ where the ground state is doubly degenerate, $E_{\sigma}(g)=0$; points $g^{*}$ and $g^{* *}$ mark the excitedstate degeneracy $E_{\mathrm{m}}=E_{\sigma}$.

ways to identify quantum phases and phase boundaries based on the symmetry and degeneracy of $E_{0}(X, Y ; 4 n)$, on excitation energies, and on correlation functions. For example, $E_{0}(X, Y ; 4 n)$ is doubly degenerate at $X_{\mathrm{c}}, Y_{\mathrm{c}}$ where one GS transforms as $\sigma=1$ and the other as -1 . We then have $E_{\sigma}\left(X_{\mathrm{c}}, Y_{\mathrm{c}} ; 4 n\right)=0$ and plus or minus linear combinations break inversion symmetry as required in a BOW phase. A magnetic gap $E_{\mathrm{m}}(X, Y ; 4 n)$ that exceeds finite-size corrections in models with equally spaced sites is another signature of a BOW phase. The GS charge distribution $\rho(X, Y ; 4 n)$ is discontinuous at $X_{\mathrm{c}}, Y_{\mathrm{c}}$ for a first order transition. We seek to identify and relate the quantum phases and phase transitions of three classes of models. Determination of phase boundaries depends on the model and almost always requires approximation.

\section{FRUSTRATED SPIN-1/2 CHAINS}

Frustrated spin-1/2 chains illustrate quantum phases more simply and directly than models with both spin and charge degrees of freedom. The Hamiltonian of the $J_{1} J_{2}$ model with $4 \mathrm{n}$ spins, PBC and isotropic exchange $J_{1}=1, J_{2}=g \geq 0$ between first and second neighbors is

$$
H(g)=\sum_{p=1}^{4 n}\left(\vec{s}_{p} \cdot \vec{s}_{p+1}+g \vec{s}_{p} \cdot \vec{s}_{p+2}\right)
$$

The $g=0$ limit is a linear HAF while $1 / g=0$ corresponds to linear HAFs on sublattices $\mathrm{A}$ and $\mathrm{B}$ of odd and even-numbered sites. We draw on extensive exact and numerical results ${ }^{17-19}$ for HAFs with $E_{\mathrm{m}}=E_{\sigma}=0$ in the infinite chain as well as on results ${ }^{20,21}$ at $g_{\mathrm{MG}}=1 / 2$. The total spin is $S=S_{\mathrm{A}}+S_{\mathrm{B}}$ and the GS is in the $S=0$ sector for any frustration $g \geq 0$. Sublattice spin is conserved only at $1 / g=0$, where $S_{\mathrm{A}}=S_{\mathrm{B}}=0$. Quite generally, the GS expectation value in the singlet sector of models with $\mathrm{PBC}$ is ${ }^{22}$

$$
\left\langle S_{\mathrm{A}}^{2}\right\rangle=\left\langle S_{\mathrm{B}}^{2}\right\rangle=-4 n \sum_{p=1}^{n}\left\langle\vec{S}_{1} \cdot \vec{S}_{2 p}\right\rangle=-4 n \sum_{p=1}^{n} C(2 p-1)
$$

Spin correlation functions $C(2 p-1) \leq 0$ between spins in opposite sublattices are negative for $g \geq 0$ and clearly vanish for noninteracting HAFs at $1 / g=0$.

Figure 2 shows the excitation energies ${ }^{22} E_{\mathrm{m}}$ and $E_{\sigma}$ as a function of frustration $g$ for 24 spins in Eq. 1. Six arrows from $g_{\mathrm{MG}}=1 / 2$ to $g_{10}$ indicate degenerate GS where $E_{\sigma}(g)=0$. The notation $g_{10}$ is related to the analytical model summarized in the Appendix, whose GS in the sectors $S_{\mathrm{A}}=S_{\mathrm{B}}=1$ and 0 are degenerate at $g_{10}$. Since ED respects all symmetries, eigenstates are even or odd under inversion. Plus and minus linear combinations of degenerate GS break symmetry and lead at $g_{\mathrm{MG}}$ to the Kekulé diagrams $|\mathrm{K} 1\rangle$ and $|\mathrm{K} 2\rangle$ in Figure 1a. We find $E_{\sigma}\left(g_{j}\right)=0$ at $n$ points $g_{j}$ in systems of $4 n$ spins up to the largest solved system of 28 spins. ${ }^{22}$ An expanded energy scale is needed to show $E_{\sigma}(g)$ in this range, and it is plausible to anticipate that the infinite chain has doubly degenerate GS with broken inversion symmetry over an interval in $g$. The $\sigma= \pm 1$ GS at $g_{10}$ are well approximated $^{22}$ by the product $|\mathrm{G}\rangle|\mathrm{G}\rangle$ of sublattices in their singlet GS and the singlet linear combination ${ }^{1}|\mathrm{~T}\rangle|\mathrm{T}\rangle$ of sublattices in the lowest triplet state. Moreover, the magnetic gap $E_{\mathrm{m}}(g)$ in Figure 2 exceeds finitesize effects in the interval $g_{\mathrm{MG}}<g<g_{10}$. The $J_{1} J_{2}$ model has a BOW phase with broken $\sigma$ symmetry and finite $E_{\mathrm{m}}$.

Finite-size effects are significant at phase boundaries, where theory indicates ${ }^{1,2}$ that $E_{\mathrm{m}}$ opens very slowly at a Kosterlitz-Thouless transition that is beyond finite models. Degenerate GS over a finite interval requires an infinite chain. Both $E_{\mathrm{m}}$ and $E_{\sigma}$ are HAF excitations at $g$ $=0$ or $1 / g=0$; they decrease approximately as $1 / N$ and converge slowly with logarithmic corrections. ${ }^{23}$ The condition $E_{\sigma}(g) \leq E_{\mathrm{m}}(g)$ is minimally required to have two singlets at lower energy than any triplet. Hence the excited-state crossings $g^{*}$ and $g^{* *}$ in Figure 2 are finite-size estimates of the boundaries of the BOW phase. The weak size dependence of $g^{*}(4 n)$ makes it possible to extrapolate ${ }^{7}$ very accurately to $g^{*}=0.2411$. The stronger size dependence of $g^{* *}(4 n)$ leads ${ }^{21,22}$ to $g^{* *} \sim 2.02(3)$.

As mentioned in connection with ionic solids and NITs, first order transitions are simple in the limit of zero overlap. The limit for spin chains is less familiar. We always have $S_{\mathrm{A}}=S_{\mathrm{B}}=0$ at $1 / \mathrm{g}=0$. The largest possible values are $S_{\mathrm{A}}=S_{\mathrm{B}}=n$ for ferromagnetic sublattices, when the singlet GS is a linear combination 


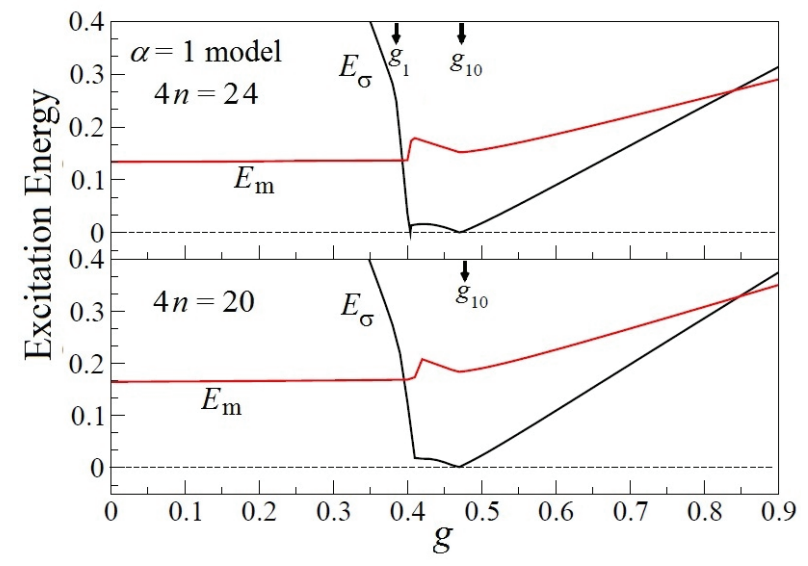

Figure 3. Excitation energies $E_{\mathrm{m}}$ and $E_{\sigma}$ as functions of frustration $g$ in models with exchanges $\alpha=1$ in Eq. 3 and $4 n=24$ or 20 spins; $E_{\mathrm{m}}$ is to the lowest triplet, $E_{\sigma}$ to the lowest singlet with reversed inversion symmetry. The ground state is doubly degenerate at $g_{10}$ where $E_{\sigma}\left(g_{10}\right)=0$ and at $g_{1}$ for 24 spins. For $g>g_{10}$, the triplet is doubly degenerate and the excited singlet is ${ }^{1}|\mathrm{~T}\rangle|\mathrm{T}\rangle$, a triplet on each sublattice.

of product functions with $S_{z}$ components $-n \leq M_{A} \leq n$ on sublattice A and $M_{\mathrm{B}}=-M_{\mathrm{A}}$ on sublattice $\mathrm{B}$. We recently constructed and solved an analytical model ${ }^{22}$ with $N=4 n$ spins, and conserved $S_{\mathrm{A}}, S_{\mathrm{B}}$. As summarized in the Appendix, the infinite chain has a first order quantum transition at $g_{c}=1 /(4 \ln 2)$ where $S_{\mathrm{A}}=S_{\mathrm{B}}$ changes discontinuously from $n$ to 1 , followed at $g_{10}=4 / \pi^{2}$ by changing from $S_{\mathrm{A}}=S_{\mathrm{B}}=1$ to 0 . All spin correlation functions $C(2 p-1)$ in Eq. 2 vanish rigorously for $g>4$ / $\pi^{2}$. The GS for $g<g_{c}$ with $S_{\mathrm{A}}=S_{\mathrm{B}}=n$ and ferromagnetic sublattices is completely different from the GS for $g>g_{10}$ with $S_{\mathrm{A}}=S_{\mathrm{B}}=0$ and antiferromagnetic sublattices. Both phases of the infinite chain rigorously have $E_{\mathrm{m}}=0$.

To discuss spin chains with nonfrustrating exchange beyond first neighbors, we retain frustrating $g$ between second neighbors and generalize ${ }^{6,22}$ the first term in Eq. 1 to

$$
H(\alpha)=\sum_{r=1}^{2 n-1} J_{r}(\alpha) \sum_{p=1}^{4 n} \vec{s}_{p} \cdot \vec{s}_{p+r}+J_{2 n}(\alpha) \sum_{p=1}^{2 n} \vec{s}_{p} \cdot \vec{s}_{p+2 n}
$$

The resulting models $H(g, \alpha)=H(\alpha)+g \Sigma_{p} \boldsymbol{s}_{p} \boldsymbol{s}_{p+2}$ have two parameters, frustration $g$ and range $1 / \alpha$ of nonfrustrating $J_{r}(\alpha)$ between spins $p$ and $p+r$. Sublattice spin is not conserved in general. The chosen distance dependence is a power law,

$$
J_{r \neq 2}(\alpha)=\frac{(-1)^{r-1}}{r^{\alpha}}\left(1+\frac{1}{2}\left(\frac{1}{2 n}\right)^{\alpha}+\sum_{s=3}^{2 n-1} \frac{1}{s^{\alpha}}\right)^{-1}
$$

with normalization $\Sigma_{\mathrm{r}}\left|J_{r}\right|=1$. The $J_{1} J_{2}$ model is the limit $1 / \alpha=0$ while constant $\left|J_{r}\right|$ is the opposite limit $\alpha=0$. At

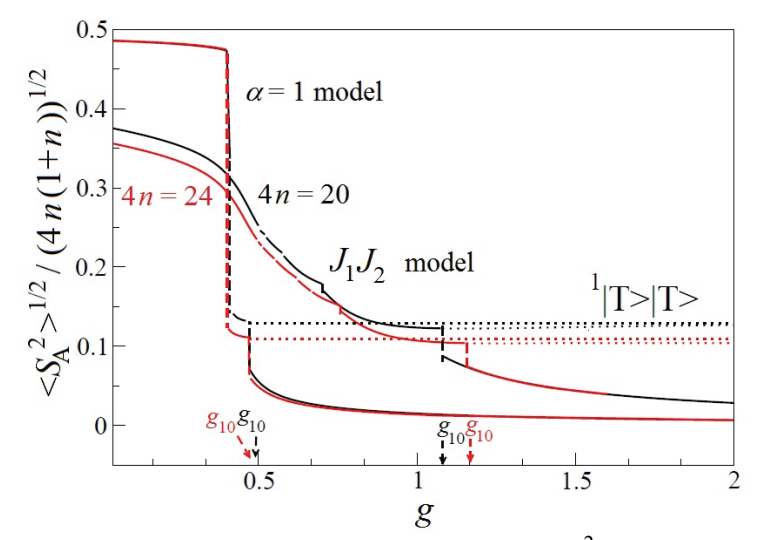

Figure 4. Ground-state sublattice spin $\left\langle S_{\mathrm{A}}{ }^{2}\right\rangle$ in Eq. 2 as a function of frustration $\mathrm{g}$ for the $J_{1} J_{2}$ and $\alpha=1$ models with 20 and 24 spins. The quantum transition of the $\alpha=1$ model is first order. The $J_{1} J_{2}$ model shows finite-size gaps; the infinite chain has continuous $\left\langle S_{\mathrm{A}}{ }^{2}\right\rangle$ per site and continuous quantum transitions. Dotted lines for $g>g_{10}$ refer to $\left\langle S_{\mathrm{A}}{ }^{2}\right\rangle$ of the excited singlet ${ }^{1}|\mathrm{~T}\rangle|\mathrm{T}\rangle$ with a triplet on each sublattice.

$g=0$, the GS has ${ }^{24}$ long-range order (LRO) for $\alpha<2$, long-range spin fluctuations but no LRO for $\alpha>2$ and there are some exact field theory results ${ }^{25}$ at $\alpha=2$.

Two points are worth noting before turning to models with fixed $\alpha$. First, $C(2 p-1) \leq 0$ holds for any $H(\alpha)$ in Eq. 3 and the $1 / g=0$ limit is always decoupled HAFs on sublattices with $S_{\mathrm{A}}=S_{\mathrm{B}}=0$. Second, each sublattice is an HAF with $2 n$ spins and PBC whose eigenstates may be classified as $0 \leq S_{\mathrm{A}}, S_{\mathrm{B}} \leq n$. Product functions $\left|r, S_{\mathrm{A}}\right\rangle\left|r^{\prime}, S_{\mathrm{B}}\right\rangle$ with $r, r^{\prime}=1,2, \ldots$ form a complete orthonormal basis at any frustration $g$. Hence the sublattice spin $\left\langle S_{\mathrm{A}}{ }^{2}\right\rangle$ in Eq. 2 always decreases with increasing $g$ and vanishes at $1 / g=0$.

Figure 3 shows the excitation energies $E_{\mathrm{m}}$ and $E_{\sigma}$ as a function of frustration $g$ in chains with exchange $\alpha$ $=1$ in Eq. 4 and LRO at $g=0$. We find $E_{\sigma}(g, 4 n)=0$ at two points, $g_{1}$ and $g_{10}$, that correspond in the analytical $(\alpha=0)$ model $^{22}$ to degeneracy between $S_{\mathrm{A}}=n$ and 1 and between $S_{\mathrm{A}}=1$ and 0 , respectively. The $\alpha=1$ model of $4 n=20$ spins, odd $n$, has $E_{\sigma}(4 n)=0$ at $g_{10}$ and an avoided crossing the $E_{\sigma}$ kink at $g_{1}$ because the GS is even under inversion at both $S_{\mathrm{A}}=n$ and 1 in the analytical model. As shown in the Appendix, the analytical model has constant $E_{\mathrm{m}}(g, 4 n)=2 /(4 n-1)$ for $g \leq g_{10}$ and doubly degenerate triplet for $g \geq g_{10}$ :

$$
E_{\mathrm{m}}(g, 4 n)=g E_{\mathrm{m}}(2 n)-2 /(4 n-1) ; g \geq g_{10}(4 n)
$$

where $E_{\mathrm{m}}(2 n)$ is the singlet-triplet gap of a $2 n$-spin HAF. Exact results account well for the $\alpha=1$ model in Figure 3: $E_{\mathrm{m}}(g)$ is almost constant for $g<g_{10}$ and the triplet is doubly degenerate for $g>g_{10}$; the slope $\mathrm{d} E_{\mathrm{m}} / \mathrm{d} g$ $=E_{\mathrm{m}}(2 n)$ holds to better than $1 \%$. Moreover, the $g>$ $g_{10}$ excitation $E_{\sigma}(g, 4 n)$ with opposite inversion symmetry is the singlet ${ }^{1}|\mathrm{~T}\rangle|\mathrm{T}\rangle$ constructed from a triplet on 


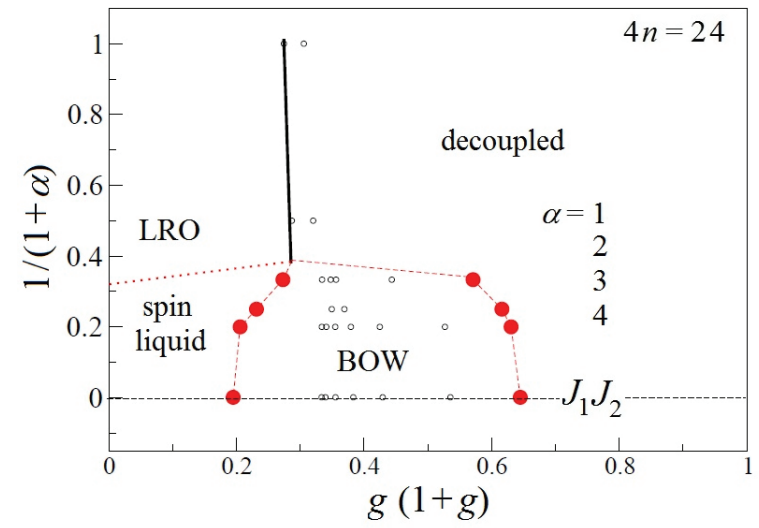

Figure 5. Quantum phase diagram $E_{0}(g, 1 / \alpha)$ of 24 spins with frustrating $J_{2}=g$ between second neighbor and nonfrustrating exchange $J_{r}(\alpha)$ in Eq. 4. Open and solid points indicate degenerate GS with $E_{\sigma}=0$ and degenerate excited states $E_{\mathrm{m}}=E_{\sigma}$, respectively. Dashed lines are approximate boundaries of the BOW phase. Solid lines are approximate boundaries of the decoupled phase in models with LRO at $g=0$; the dotted line separates models ${ }^{6}$ with long-range fluctuations and order at small frustration.

each sublattice. The slope $\mathrm{d} E_{\sigma} / \mathrm{d} g$ in Figure 3 is within a few percent of $2 E_{\mathrm{m}}(2 n)$, the exact result in Appendix. The $\alpha=1$ model has LRO at $g=0$, a first order quantum transition at $g_{1}$ to the decoupled phase, and $E_{\mathrm{m}}=E_{\sigma}$ $=0$ in the infinite chain over the entire range $g \geq 0$.

The evolution of $\left\langle S_{\mathrm{A}}^{2}\right\rangle^{1 / 2} /[4 n(n+1)]^{1 / 2} \leq 0.50$ with frustration $\mathrm{g}$ in Figure 4 highlights the difference between models with first order and continuous quantum transitions. The $\alpha=1$ model has almost ferromagnetic sublattices at $\mathrm{g}=0$ where the $J_{1} J_{2}$ model has smaller $\left\langle S_{\mathrm{A}}{ }^{2}\right\rangle$ associated with the HAF and long range spin fluctuations. LRO at $g=0$ leads to a first order transition and fundamentally different GS on either side as seen clearly in the analytical model. The models have similar $\left\langle S_{\mathrm{A}}{ }^{2}\right\rangle$ at large frustration $g>g_{10}$ where dotted lines refer to ${ }^{1}|\mathrm{~T}\rangle|\mathrm{T}\rangle$ with $S_{\mathrm{A}}{ }^{2}=2$ at $1 / g=0$. The $g=0$ behavior is not relevant in the decoupled phase. The excited state of either model at $g_{10}$ is already within $5 \%$ of the $1 / g=0$ limit of decoupled sublattices. At still larger frustration $g$, the lowest singlet excitation is doubly degenerate, $|\mathrm{A}\rangle|\mathrm{G}\rangle$ or $|\mathrm{G}\rangle|\mathrm{A}\rangle$, where $|\mathrm{A}\rangle$ is the lowest singlet excited state of the sublattice.

The quantum phase diagram for frustrated spin chains in Figure 5 is based ${ }^{22}$ on models with 24 spins and $\alpha=1-4$ in Eq. 4 in addition to the $J_{1} J_{2}$ model. Open symbols refer to GS degeneracy with $E_{\sigma}(g)=0$, closed symbols to $E_{\sigma}(g)=E_{\mathrm{m}}(g)$ in models with continuous quantum transitions and a BOW phase. We find closely similar phase diagrams for 20 or 16 spins. $^{22}$ Models with LRO at $g=0$ have a first order transition to a decoupled phase. The BOW phase narrows with increasing range $1 / \alpha$ of nonfrustrating exchange and shrinks to a multicritical point ${ }^{6,22}$ around $\alpha_{t} \sim 1.8, g_{t} \sim 0.4$. The same pattern is found below in systems with charge as well as spin excitations. Some parameters return continuous quantum transitions and an intermediate BOW phase; other parameters lead to a first order quantum transition and no BOW phase.

\section{COMPETING INTERACTIONS IN QUANTUM CELL MODELS}

1D models with both spin and charge degrees of freedom have richer quantum phase diagrams than spin chains. We are considering half-filled bands with inversion symmetry $\sigma$ at sites, equally spaced sites, conserved total spin and singlet GS. Gaps associated with adding or subtracting an electron discriminate between insulators and conductors; such gaps are not needed here. Models with a BOW phase have $E_{\sigma}=0$ and two continuous transitions associated with $E_{\mathrm{m}}$ and $E_{\sigma}$. Both gaps open at a first order transition.

The EHM has on-site repulsion $U \geq 0$ for two electrons in the same Wannier orbital, repulsion $\mathrm{V}$ for electrons on adjacent sites and electron transfer $t=1$ between adjacent sites. The Hamiltonian with PBC is

$$
\begin{gathered}
H_{\mathrm{EHM}}(U, V)=-\sum_{p=1, \sigma}^{4 n}\left(a_{p, \sigma}^{+} a_{p+1, \sigma}+\text { h.c. }\right)+ \\
U \sum_{p=1}^{4 n} n_{p}\left(n_{p}-1\right) / 2+V \sum_{p=1}^{4 n} n_{p} n_{p+1}
\end{gathered}
$$

where $a_{p \sigma}^{+}\left(a_{p \sigma}\right)$ creates (annihilates) an electron with spin $\sigma$ in the orbital at site p, h.c. is the hermitian conjugate and $n_{\mathrm{p}}$ is the number operator. Half-filled bands have one electron per site. There are four simple limits: $U=V=0$ is a Hückel or tight-binding band of width $4 t$ that is readily solved for any size; $U>>V 0$ has singly occupied sites, $n_{p}=1$, and magnetic properties given by an HAF with $J_{1}=4 t^{2} /(U-V)$; the GS for large $V$ is a charge density wave (CDW) with doubly occupied sites on one sublattice and empty sites on the other; when both $U$ and $V$ are large, there is a first-order quantum transition at $V \sim U / 2$ between spin liquid and CDW. Hirsch $^{16}$ found the $t^{2} /(U-V)$ correction to the phase boundary and van Dongen ${ }^{15}$ the $t^{4}$ correction. Nakamu$\mathrm{ra}^{1}$ used symmetry, field theory and ED to establish the existence of a BOW phase $U<U_{t}$, the tricritical point that has subsequently been independently estimated ${ }^{3-5}$ as $U_{t} \sim 6.7$ for the EHM.

The EHM has electron-hole (e-h) symmetry ${ }^{26}$ $J= \pm 1$ that corresponds to a phase factor and interchanged empty and doubly occupied sites. E-h symmetry holds in half-filled bands with any sitediagonal interaction instead of $V$ in Eq. 6, including 3D interactions or two-center integrals in the Pariser-ParrPople (PPP) model of conjugated molecules. But of 


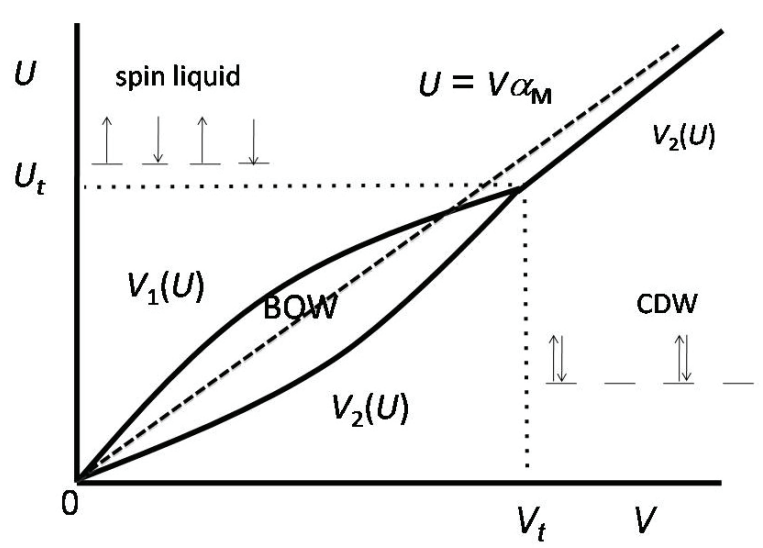

Figure 6. Quantum phase diagram of extended Hubbard models, Eq. 6, with on-site $U>0$, nearest-neighbor $V>0$, and 1D potential with Madelung constant $\alpha_{\mathrm{M}}$. The dashed line is the first order transition from the spin liquid to the CDW phase in the limit of zero overlap. In units of $t=1$, the tricritical point $U_{t}, V_{t}$ separates first order and continuous transitions. The line $V_{2}(U)$ is a first order transition from spin liquid to CDW for $U>U_{t}$ and a continuous transition from BOW to a CDW for $U<U_{t}$. The BOW phase between $V_{2}(U)$ and $V_{1}(U)$ has a finite magnetic gap, $E_{\mathrm{m}}$, that opens at $V_{1}$ and vanishing gap $E_{\sigma}$ that opens at $V_{2}$.

course $U_{t}$ depends on the chosen potential. ${ }^{5}$ The crucial requirement for e-h symmetry is electron transfer between sites in opposite sublattices, the first term in Eq. 6. The following discussion holds for other quantum cell models than the EHM. According to the HellmannFeynman theorem, the density of doubly occupied sites is

$$
\rho_{2}(U, V)=\left(\frac{\partial E_{0}(U, V)}{\partial U}\right)_{V}
$$

Charge conservation leads to the same density of empty sites in half-filled bands and to $1-2 \rho_{2}$ for the density of singly occupied sites. Increasing $V$ at constant $U_{c}>U_{\mathrm{t}}$ generates a first order quantum transition at $E_{\sigma}\left(U_{\mathrm{c}}, V_{\mathrm{c}}\right)=0$. The partial derivative is more directly related to a quantum phase transition than the sublattice spin $\left\langle S_{\mathrm{A}}{ }^{2}\right\rangle$ in Eq. 2. However, since only singly occupied sites contribute to $S_{\mathrm{A}}$, discontinuous $\rho_{2}$ clearly implies discontinuous $\left\langle S_{\mathrm{A}}{ }^{2}\right\rangle$.

The schematic quantum phase diagram ${ }^{1-5}$ in Figure 6 holds for the EHM and for models with spinindependent Coulomb interactions. If we suppose that singly occupied sits are neutral, then empty sites are positively charged and doubly occupied sites are negatively charged. The electrostatic energy of the ionic lattice is $E_{\mathrm{M}}=\alpha_{\mathrm{M}} V$ per ion pair, where $V$ is the Coulomb interaction between neighbors and $\alpha_{\mathrm{M}}$ is the
Madelung constant; $\alpha_{\mathrm{M}}=2$ for the EHM, $2 \ln 2$ for a 1D array of point charges and $\alpha_{M}$ can be evaluated for 3D lattices. The dashed line in Figure 6 is the limit of zero overlap that separates the spin liquid phase, a Curie spin system at $t=0$, from the CDW phase. We notice similarities and differences to frustrated spin chains in Figure 5 . Finite $N=4 n$ systems have a single point $E_{\sigma}\left(U_{c}\right.$, $\left.V_{c}, 4 n\right)=0$, as $\mathrm{do}^{5} N=4 n+2$ systems with antiperiodic (twisted) BC. The quantum transition is first order for $U_{\mathrm{c}}>U_{t}$ as estimated, for instance, from discontinuous $E_{0}$ derivatives. In contrast to the $J_{1} J_{2}$ model with multiple GS degeneracies in Figure 2, the width of the BOW phase for $U_{\mathrm{c}}<U_{t}$ in Figure 6 is entirely based on excited-state degeneracies. The spin gap opens at $E_{\mathrm{m}}\left(U_{\mathrm{c}}, V_{1}, 4 n\right)=E_{\sigma}\left(U_{\mathrm{c}}, V_{1}, 4 n\right)$ with $V_{1}<U_{\mathrm{c}} / \alpha_{\mathrm{M}}$. The inversion gap opens at $E_{\sigma}\left(U_{\mathrm{c}}, V_{2}, 4 n\right)=E_{\mathrm{J}}\left(U_{\mathrm{c}}, V_{2}, 4 n\right)$ with $V_{2}>U_{\mathrm{c}} / \alpha_{\mathrm{M}}$ where $E_{\mathrm{J}}$ is the excitation energy of the lowest singlet with opposite e-h symmetry to the GS. Finite $E_{\mathrm{m}}\left(U_{\mathrm{c}}, V_{\mathrm{c}}\right)$ for $U_{\mathrm{c}}<U_{t}$ can be estimated ${ }^{5}$ by extrapolation of ED results or by density matrix renormalization group (DMRG) calculations. Although not as decisively as in Figure $2, E_{\mathrm{m}}\left(U_{\mathrm{c}}, V_{\mathrm{c}}\right)$ exceeds ${ }^{5}$ finite-size effects for intermediate $U$ and supports to the existence of a BOW phase that narrows at small $U, V$ and shrinks to a point at $U=V=0$ in Figure 6 . The BOW phase near the band limit has been established by other theoretical methods ${ }^{27}$ and is beyond the scope of the present discussion.

To pursue the analogy with frustrated spin chains, we note that $\left\langle S_{\mathrm{A}}{ }^{2}\right\rangle$ increases with $U$ at $V=0$ and decreases with $V$ at constant $U$. The largest possible $\left\langle S_{\mathrm{A}}{ }^{2}\right\rangle$ at $U>>\mathrm{V}=0$ is just that of an HAF, the $J_{1} J_{2}$ model at $g=0$. The GS has long range spin fluctuations but no LRO. The same limit holds for $U>\alpha_{\mathrm{M}} V$ and $U-V>>t$. We always have $\left\langle S_{\mathrm{A}}{ }^{2}\right\rangle=0$ at large $V$ where the GS is a CDW, doubly degenerate in e-h symmetry, and both $E_{\mathrm{m}}$ and $E_{\sigma}$ are of order $V-U$. Although the CDW is doubly degenerate, all linear combinations transform as $\sigma=1$ and two singly occupied sites are minimally needed for either a triplet or a singlet state that is odd under inversion. Systems with a BOW phase again have two continuous quantum phase transitions while systems with a first order transition do not support a BOW phase.

Organic CT crystals contain ${ }^{11,12}$ mixed 1D stacks of planar $\pi$-electron donors (D) and acceptors (A). There are two sites per unit cell. Inversion symmetry is retained on the neutral side but is broken on the ionic side by the Peierls instability to dimerization. The present discussion is limited to models with equal spacing in Figure 1b. As sketched there for zero overlap, the neutral stack is a CDW with both electrons in the HOMO of $\mathbf{D}$ while the ionic stack has one electron on each $\mathbf{D}^{+}$and $\mathbf{A}^{-}$. We have reversed spin-charge relations with paired spins in the neutral limit and $s=1 / 2$ sites in the ionic limit. It is convenient and physically 
sensible to focus on neutral and singly ionized sites with spin $\alpha$ or $\beta$, but to exclude doubly ionized $\mathbf{D}^{2+}$ or $\mathbf{A}^{2-}$. If we take the $\mathbf{D}$ and $\mathbf{A}$ sublattices to have odd and evennumbered sites, respectively, the restricted basis has charge operators $q_{\mathrm{p}}$ which assume the values 0,1 at odd $p$ for $\mathbf{D}, \mathbf{D}^{+}$and $q_{p}=0,-1$ at even $p$ for $\mathbf{A}, \mathbf{A}^{-}$.

The CT models has site energies $\pm \Gamma$ at $\mathbf{A}$ and $\mathbf{D}$ sites and Coulomb interactions $V_{p p}$, between ions in the stack or the $3 \mathrm{D}$ crystal at the mean field level. The Hamiltonian in the restricted basis with $t=1$ is

$$
\begin{aligned}
& H_{\mathrm{CT}}(\Gamma, V)=\Gamma \sum_{p=1}^{4 n}(-1)^{p} n_{p}- \\
& \sum_{p=1, \sigma}^{4 n}\left(a_{p, \sigma}^{+} a_{p+1, \sigma}+\text { h.c. }\right)+\sum_{p>p^{\prime}=1}^{4 n} V_{p p^{\prime}} q_{p} q_{p^{\prime}}
\end{aligned}
$$

The charge operator $q_{p}$ is $2-n_{p}$ at $\mathbf{D}$ sites and $-n_{p}$ at $\mathbf{A}$ sites. Hence $V_{p p},>0$ corresponds to attraction for ions in different sublattices and repulsion for ions in the same sublattice. The special case $H_{\mathrm{CT}}(\Gamma, 0)$ is the modified Hubbard model (MHM) whose continuous NIT has been treated in detail. ${ }^{28}$ The GS electron density $n_{\mathbf{D}}(\Gamma, V)$ per site is

$$
n_{\mathbf{D}}(\Gamma, V)=1-\left(\frac{\partial E_{0}(\Gamma, V)}{\partial \Gamma}\right)_{V}
$$

The GS energy per site is $-\Gamma$ for a neutral lattice in Figure $1 \mathrm{~b}$ for $\Gamma \gg>$. The bond order between site $\mathrm{m}$ and $m+1$ is the GS expectation value

$$
p_{\mathrm{m}}=-\frac{1}{2}\left(\frac{\partial E_{0}(\Gamma, V, t)}{\partial t_{\mathrm{m}, \mathrm{m}+1}}\right)_{\Gamma, V}=\left\langle\left(a_{\mathrm{m}, \alpha}^{+} a_{\mathrm{m}+1, \alpha}+a_{\mathrm{m}, \beta}^{+} a_{\mathrm{m}+1, \beta}\right)\right\rangle
$$

PBC returns equal bond orders for nondegenerate GS. Linear combinations of doubly degenerate GS lead to BOWs with amplitude $\pm B$. The ionic limit $\Gamma<<0$ is again an HAF. Virtual transfers in the ionic limit are reduced by a factor of two in the restricted basis, thereby reducing $J_{1}$, but are not reduced in the neutral limit. Hence the MHM phase boundary ${ }^{29}$ at $\Gamma=0$ for $t=0$ shifts to $\Gamma<0$ for finite $t$ while the EHM phase boundary $^{16}$ at $V=U / 2$ for $t=0$ shifts to $V>U / 2$ for finite $t$.
The MHM has a continuous ${ }^{28}$ NIT at $\Gamma_{\mathrm{c}}=-0.666$ where the ionicity $\rho\left(\Gamma_{\mathrm{c}}\right)=0.684$ is the fraction of an electron transferred from $\mathbf{D}$ to $\mathbf{A}$. The dipole-allowed CT excitation is $E_{\sigma}$ on the neutral side. We have $\mathrm{E}_{\sigma}=0$ for $\Gamma<\Gamma_{\mathrm{c}}$ and very little oscillator strength for what becomes a spin excitation on the ionic side. Table 1 lists $\Gamma_{\mathrm{c}}(4 n)$ where the GS is doubly degenerate, $E_{\sigma}=0$, the BOW amplitude $\mathrm{B}\left(\Gamma_{\mathrm{c}}, V\right)$ and $\Gamma_{\sigma}(4 n)$ where the excited states are degenerate, $E_{\mathrm{m}}=E_{\sigma}$. We start with the MHM model and return below to $V=0.3$. The magnetic gap opens at $\Gamma_{\sigma} ; E_{\mathrm{m}}$ exceeds finite-size effects ${ }^{28}$ at $\Gamma_{\mathrm{c}}$; and the size dependence of $\Gamma_{\sigma}$ or $\Gamma_{\mathrm{c}}$ is weak. The width of the BOW phase is $\Gamma_{\sigma}-\Gamma_{\mathrm{c}} \sim 0.30$ in units of $t$. The BOW amplitude at $\Gamma_{\mathrm{c}}$ is large, $B=0.139$ for 20 spins, comparable to the mean value, $\left(p_{1}+p_{2}\right) / 2=0.377$. For comparison, the BOW amplitude of the Kekule structures in Figure 1a is $B=3 / 8$, as follows from $\left(\left\langle\mathbf{s}_{1} \cdot \mathbf{s}_{2}\right\rangle-\left\langle\mathbf{s}_{2} \cdot \mathbf{s}_{3}\right\rangle\right) / 2$ $= \pm 3 / 8$.

The MHM has strong electron correlation enforced by the restrictions $n_{\mathrm{p}}=1$ or 2 at $\mathbf{D}$ sites and $n_{\mathrm{p}}=$ 0 or 1 at $\mathbf{A}$ sites. The model is not cooperative, however, since there is no dependence on the number of ions. Coulomb interactions $V_{p p}$, in Eq. 8 between $\mathbf{D}^{+}$and $\mathbf{A}^{-}$ sites are attractive and cooperative, preferentially stabilizing more and more ions. Either by exact solution of finite stacks or in mean-field theory of $1 \mathrm{D}$ or 3D lattices, increasing Coulomb interactions lead ${ }^{13,12}$ to a first order NIT. The continuous NIT of the MHM with $E_{0}(\Gamma, 0)$ and equal spacing becomes first order in models with $E_{0}(\Gamma, V)$ and $V>V_{t}$, at a tricritical point that depends on $V_{p p}$. Just as for spin chains in Figure 5 or for extended Hubbard models in Figure 6, the $\Gamma, V$ quantum phase diagram of $H_{\mathrm{CT}}(\Gamma, V)$ has a BOW phase at $V=0$ between a spin liquid for $\Gamma<\Gamma_{\sigma}$ and a CDW for $\Gamma>\Gamma_{\mathrm{c}}$. The BOW phase narrows and disappears for $V>V_{\mathrm{t}}$. The first order NIT at $\Gamma_{\mathrm{c}}, V_{\mathrm{c}}$ for $V_{\mathrm{c}}>V_{t}$ is directly from a spin liquid with $E_{\mathrm{m}}=E_{\sigma}=0$ to a $\mathrm{CDW}$ with finite spin and charge gaps.

If attractive interactions in $H_{\mathrm{CT}}(\Gamma, V)$ induce a first order transition, repulsive interactions should broaden the BOW phase. With this motivation, we chose $V_{p p}=V \delta_{\mathrm{p}, \mathrm{p}+2}$ in Eq. 8 with $V>0$ for repulsion between adjacent ions in the same sublattice. As seen in Table 1, small $V=0.3$ hardly changes the BOW amplitude $B\left(\Gamma_{\mathrm{c}}, V\right)$ at the GS degeneracy while noticeably increasing the width $\Gamma_{\sigma}-\Gamma_{\mathrm{c}}$ of the BOW phase. The

Table 1. Ground and excited state degeneracy $E_{\sigma}=0$ and $E_{\sigma}=E_{\mathrm{m}}$ at $\Gamma_{\mathrm{c}}$ and $\Gamma_{\sigma}$, respectively, of $H_{\mathrm{CT}}$ with $4 n$ sites in Eq. 8; $B$ is the BOW amplitude at $\Gamma_{\mathrm{c}} ; V=0.3$ refers to repulsion between second neighbor ions.

\begin{tabular}{ccccccc}
\hline Size & \multicolumn{3}{c}{ MHM $(V=0)$} & & \multicolumn{3}{c}{0.3} \\
\hline$N=4 n$ & $\Gamma_{\sigma}$ & $\Gamma_{\mathrm{c}}$ & $B\left(\Gamma_{\mathrm{c}}, 0\right)$ & $\Gamma_{\sigma}+V$ & $\Gamma_{\mathrm{c}}+V$ & $B\left(\Gamma_{\mathrm{c}}, V\right)$ \\
12 & -1.018 & -0.644 & 0.1707 & -1.143 & -0.724 & 0.1717 \\
16 & -0.994 & -0.654 & 0.1516 & -1.119 & -0.736 & 0.1526 \\
20 & -0.972 & -0.658 & 0.1387 & -1.112 & -0.740 & 0.1398 \\
\hline
\end{tabular}


same trends are observed with increasing $V$ until large $V$ induces charge disproportionation in each sublattice. The GS in the limit $V>>2$ is a four-fold degenerate CDW-BOW of neutral and ionic dimers, $\left(\mathbf{D A} \mathbf{A D}^{+} \mathbf{A}^{-}\right)_{n}$ or $\left(\mathbf{A D A}^{-} \mathbf{D}^{+}\right)_{n}$, with interchanged molecules and ions in the other two GS. Singlet pairing of radical ions leads to finite $E_{\mathrm{m}}$.

The normalized sublattice spin $\left\langle S_{\mathrm{A}}{ }^{2}\right\rangle^{1 / 2} /[4 n \cdot(n+$ $1)]^{1 / 2}$ is shown in Figure 7 for $H_{\mathrm{CT}}(\Gamma, 0)$ as a function of $\Gamma$ for $4 n=12,16$ and 20. The infinite chain has continuous $\left\langle S_{\mathrm{A}}{ }^{2}\right\rangle$, as seen by extrapolation of the finite-size jumps at $E_{\sigma}\left(V_{\mathrm{c}}, 0,4 n\right)$. The HAF result of 0.412 for 16 spins and 0.378 for 20 spins is the $\Gamma \rightarrow-\infty$ limit, some $10 \%$ higher than at $\Gamma=-2$. The HAF is also the $g=0$ point in Figure 4 for the $J_{1} J_{2}$ chain or the $U>V$ limit of extended Hubbards models. The neutral lattice at $\Gamma>>0$ has $S_{\mathrm{A}}=S_{\mathrm{B}}=0$ for filled and empty sublattices.

We close this Section with some remarks about the ionic Hubbard model (IHM) with site energy $\pm \Delta$ on the sublattices of even and odd sites, ${ }^{30,31}$

$$
\begin{aligned}
& H_{I H M}(U, \Delta)=-t \sum_{p, \sigma}\left(a_{p, \sigma)}^{+} a_{p+1, \sigma)}+\text { h.c. }\right) \\
& +U \sum_{p} n_{p}\left(n_{p}-1\right) / 2+\Delta \sum_{p}(-1)^{p} n_{p}
\end{aligned}
$$

The IHM has a BOW phase ${ }^{30}$ and its quantum phase diagram in the $(U, \Delta)$ plane has been discussed ${ }^{31}$ in detail mainly in terms of DMRG. The IHM is closely related to $H_{C \mathrm{~T}}(\Gamma, V)$ and illustrates the different contexts and motivation in which the models were studied. The IHM is a Hubbard model at $\Delta=0$, an insulator at $U=0$ with energy gap $2 \Delta$ between the valence and conduction bands, and a CDW in the limit $\Delta>U, t$. It has simple familiar limits and is ideal for exploring quantum phases. The IHM always has two continuous quantum phase transitions: ${ }^{31}$ With $t=1$ as usual and constant $\Delta$, both $E_{\mathrm{m}}$ and $E_{\sigma}$ are finite for $U<U_{\mathrm{cl}}$, a first critical point beyond which $E_{\sigma}=0 ; E_{\mathrm{m}}$ goes to zero at a second critical point $\mathrm{U}_{\mathrm{c} 2}$; the BOW phase is the interval $\left[U_{\mathrm{c} 1}, U_{\mathrm{c} 2}\right]$. Two continuous transitions and a BOW phase are fully consistent with the preceding discussion of $H_{\mathrm{CT}}(\Gamma, 0)$. The IHM cannot support a first order transition that requires cooperative attractive interactions since Eq. 11 has no interactions $V_{p p}$, between ions.

In the context of CT crystals, on the other hand, large $3 \mathrm{D}$ electrostatic interactions were recognized ${ }^{10-12}$ from the outset to be necessary to stabilize the ionic phase and drive neutral ionic transitions. Interactions $V_{p p}$, can be treated explicitly in 1D models or at the mean field level with Madelung sums over $\left\langle V_{p p}\right\rangle$, and preceded the basic model $H_{\mathrm{CT}}(\Gamma, 0)$. Both on physical grounds and to reduce the number of parameters as well as the size of the basis, the $\Delta$ and $U$ parameters in Eq. 11 were combined into the site energy $\Gamma$ defined $^{9}$ as

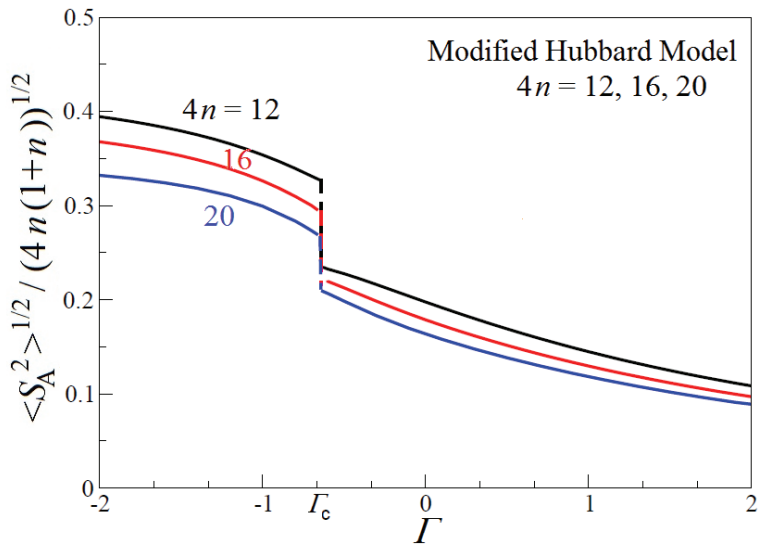

Figure 7. Ground-state sublattice spin $\left\langle S_{\mathrm{A}}{ }^{2}\right\rangle$ in Eq. 2 as a function of site energy $\Gamma$ in the modified Hubbard model, Eq. 7 with $V=0$, for 12,16 and 20 sites. The infinite stack has continuous $\left\langle S_{\mathrm{A}}{ }^{2}\right\rangle$ per site while finite stacks are discontinuous at $\Gamma_{\mathrm{c}}$. In the limit $\Gamma \rightarrow-\infty$ of a linear HAF, $\left\langle{S_{\mathrm{A}}}^{2}\right\rangle$ coincides with $g=0$ of the $J_{1} J_{2}$ model in Figure 4.

$\Delta-U / 2$ in the limit $\Delta, U \rightarrow \infty$ such that the difference is finite. The limit gives the restricted basis for $H_{\mathrm{CT}}(\Gamma, V)$. As discussed elsewhere, ${ }^{13,32}$ the model with coupling to vibrations describes IR, optical and dielectric properties of CT crystals with continuous or firstorder neutral ionic transitions.

\section{DISCUSSION}

We have explored similarities among the quantum phase diagrams of three classes of 1D models with equally spaced sites: frustrated spin chains with variable range exchange, half-filled Hubbard models with spinindependent interactions and ionic or modified Hubbard models with site energies. These models have been studied separately in quite different contexts. We would like to understand why models with BOW phases have similar quantum phase diagrams and phase transitions.

The common thread is the spin-1/2 linear Heisenberg antiferromagnet (HAF). The HAF is the narrow band limit $4 t<<U-V$ of the EHM in Eq. 6 or of related quantum cell models; it is the $\Gamma<<0$ limit of the MHM or related models in Eq. 8; it is the limit $1 / g=0$ of frustrated spin chains with arbitrary range $1 / \alpha$ of nonfrustrating exchange; and of course it is the $g=0$ chain in Eq. 1. The HAF conserves total spin $S$ but not the sublattice spin $S_{\mathrm{A}}, S_{\mathrm{B}}$ of odd and even numbered sites. The infinite HAF is rigorously known to have a nondegenerate GS and $E_{\mathrm{m}}=E_{\sigma}=0$. The GS expectation value $\left\langle S_{\mathrm{A}}{ }^{2}\right\rangle / 4 n$ per spin in Eq. 2 is of order $\ln (4 n)$ due to long range spin correlations. The analytical spin chain ${ }^{22}$ in the Appendix has perfect LRO and $S_{\mathrm{A}}{ }^{2}=n(n+1)$ for $g<1 /(4 \ln 2)$. Increasing frustration $g$ always reduces $\left\langle S_{\mathrm{A}}{ }^{2}\right\rangle$ and leads to $S_{\mathrm{A}}=S_{\mathrm{B}}=0$ at $1 / g=0$. Increasing $V$ in 
Hubbard models with $U>4 t$ or $\Gamma$ in the MHM also reduces $\left\langle S_{\mathrm{A}}{ }^{2}\right\rangle$ as shown in Figure 7. Sublattice spin changes discontinuously in a first order transition from spin liquid to $\mathrm{CDW}$ as can readily be seen from the limit of no overlap. In either case, we have $S_{\mathrm{A}}=S_{\mathrm{B}}=0$ in the limit of large $V$ or large $\Gamma$, when one sublattice is filled and the other one is empty.

These models all have inversion symmetry $\sigma$ at sites. The GS of a $4 n$-spin HAF in the sector $S \leq 2 n$ alternates between $\sigma= \pm 1$ with increasing $S$. Quite generally, the GS symmetry changes at least once with increasing $g$ in spin chains, with increasing $V$ in Hubbard models or with increasing $\Gamma$ in CT models. The degeneracy $E_{\sigma}\left(X_{c}, Y_{c}\right)=0$ marks a first order quantum transition when a partial derivative of $E_{0}$, the charge density $\rho_{2}(U, V)$ in Eq. 7 or $n_{\mathrm{D}}(\Gamma, V)$ in Eq. 9, is discontinuous at the transition. $\left\langle S_{\mathrm{A}}{ }^{2}\right\rangle$ is discontinuous whenever $\rho_{2}$ or $n_{\mathrm{D}}$ is and $\left\langle S_{\mathrm{A}}{ }^{2}\right\rangle$ is discontinuous in spin chains with LRO at $g=0$. The discontinuity refers to the infinite system rather than to small jumps that decrease with increasing size.

The degeneracy $E_{\mathrm{\sigma}}\left(X_{\mathrm{c}}, Y_{\mathrm{c}}\right)=0$ indicates a BOW phase when $\rho_{2}(U, V)$ or $n_{\mathrm{D}}(\Gamma, V)$ is continuous and the finite gap $E_{\mathrm{m}}\left(X_{\mathrm{c}}, Y_{\mathrm{c}}\right)$ to the lowest triplet exceeds finitesize effects. Quite remarkably, frustrated spin chains in Figure 5 have multiple points at which $E_{\sigma}=0$ for models with $\alpha \geq 2$ and no LRO at $g=0$. The continuous phase transition is then estimated from the excited state degeneracy $E_{\sigma}\left(X_{\mathrm{c}}, Y_{\mathrm{c}} ; 4 n\right)=E_{\mathrm{m}}\left(X_{\mathrm{c}}, Y_{\mathrm{c}} ; 4 n\right)$ where $E_{\mathrm{m}}$ opens in the infinite system. A finite energy gap has major implications at absolute zero: the BOW phase is diamagnetic rather than paramagnetic and its specific heat decreases exponentially rather than linearly as $\mathrm{T} \rightarrow 0$.

We have exclusively discussed spin or electronic excitations. Reference to possible experimental realizations must take into account lattice and molecular vibrations as well as 3D interactions. Electron-phonon (e-ph) or electron-molecular-vibration (e-mv) coupling has important consequences in quasi-1D materials. ${ }^{33,34}$ Linear coupling such as $(\mathrm{d} t / \mathrm{d} u)_{0}$ or $(\mathrm{d} J / \mathrm{d} u)_{0}$ about equilibrium plus a harmonic potential generates models with Peierls or spin-Peierls transitions. The adiabatic or Born-Oppenheimer approximation then leads to electronic models with additional parameters such as the dimerization amplitude and higher-dimensional phase diagrams that are beyond the scope of this paper. Since dimerization breaks inversion symmetry, totally symmetric molecular vibrations seen in Raman spectra for equally spaced sites become strongly infrared allowed by borrowing intensity from the optical CT transition. ${ }^{35}$ The Peierls instability of $1 \mathrm{D}$ systems to dimerization becomes conditional in CT salts. ${ }^{12,13}$ NITs are quantum phase transitions since $T_{\mathrm{c}}<100 \mathrm{~K}$ is well below any electronic excitation. Spin-Peierls transitions, ${ }^{36,37}$ by contrast, are thermally driven in systems with $J_{1} \sim 10 \mathrm{~K}$.
We have remarked that the three classes of models have appeared in different contexts to address other issues than mentioned here. Dozens of organic CT crystals have either first order or continuous NITs. ${ }^{12,13}$ Tetrathiofulvalene-Chloranil (TTF-CA) has received the most attention, but related families have been well characterized and there are many donor-acceptor complexes. TTF-CA has a first order dimerization transition on cooling and is consequently not a BOW candidate. TTFBromanil has a thermal Peierls transition ${ }^{38}$ with significant Boltzmann population of spin states at low temperature. Some recurring NIT themes are to obtain crystal structures, including 3D changes, to measure and model polarized optical spectra, including pump-probe experiments, to model coupling to lattice phonons and to molecular vibrations, and to characterize elementary excitations such as spin solitons or charged polarons. Organic CT salts are semiconductors with challenging optical and magnetic properties that have been modeled, to the best of our knowledge, without invoking a BOW phase.

Quantum cell models such as the EHM or PPP model are approximate many-body theories that apply to solids or molecules in general. Hubbard models initially addressed the formation of local moments in narrow bands. The models have also been studied theoretically as interesting many-body problems, and the BOW phase was identified ${ }^{1}$ in the EHM. We recently proposed that Rb-TCNQ(II), the second polymorph of alkali-tetracyanoquinodimethane salt, is a realization of a BOW phase. ${ }^{39}$ The anion radical stacks at 100 and $300 \mathrm{~K}$ are equally spaced, ${ }^{40}$ not dimerized at low temperature. The magnetic gap is large, $E_{\mathrm{m}}>400 \mathrm{~K}$, thereby suppressing excitations ${ }^{40}$ below $200 \mathrm{~K}$, and the optical gap is even larger. Yet the strong, characteristic coupling of CT fluctuations to molecular vibrations in the mid infrared clearly indicates broken inversion symmetry below $200 \mathrm{~K}$ that cannot be due to structural dimerization.

The magnetic properties of spin chains of transition metal ions or of organic radicals have been interpreted as HAFs for many years. Generalizations include larger spin than $s=1 / 2$, anisotropic and/or antisymmetric exchange, and weak exchange between chains. Recent interest has focused in frustrated systems such as copper oxides ${ }^{41}$ with chains of spin- $1 / 2 \mathrm{Cu}$ (II) ions with either antiferromagnetic $J_{1}>0$ or ferromagnetic $J_{1}<0$ between neighbors and presumed $J_{2}>0$ between second neighbors. Exotic GS can be realized ${ }^{42}$ in $J_{1} J_{2}$ models in an applied magnetic field as observed in saturation magnetization at low temperature, or due to deviations from isotropic exchange. Spin-phonon coupling and magnetic interactions between chains also come into play. We are not aware of decisive evidence for a BOW phase system but the field is active and such a material 
may be identified. Very low temperature is needed to freeze out spin excitations. An even greater challenge is to demonstrate a phase transition to a BOW phase.

\section{CONCLUSION}

In summary, we have discussed the quantum phase diagrams and transitions of three classes of 1D models with singlet GS and inversion symmetry at sites. In some parameter sectors, the models have a first order transition where the sublattice spin $\left\langle S_{\mathrm{A}}{ }^{2}\right\rangle$ is discontinuous. In other parameter ranges, the models have two continuous transitions, continuous evolution of $\left\langle S_{\mathrm{A}}{ }^{2}\right\rangle$ and an intermediate BOW phase with a doubly degenerate GS and a finite gap $E_{\mathrm{m}}$ to the lowest triplet state. Our principal result is the similar quantum phase diagrams of spin chains with frustrating second-neighbor exchange and variable range nonfrustrating exchange, of half-filled Hubbard models with spin independent interactions, and of modified Hubbard models with site energies $\pm \Gamma$ in a restricted basis. All three classes of models reduce in some limit to a linear spin-1/2 Heisenberg antiferromagnet.

Acknowledgements. We thank the National Science Foundation for partial support of this work through the Princeton MRSEC (DMR-0819860). MK thanks S. N. Bose centre for partial financial support, and SR thanks DST India for support through various projects.

\section{APPENDIX: ANALYTICAL SPIN CHAIN WITH FRUSTRATION}

We consider a chain of $4 n$ spin with $\mathrm{PBC}$ and uniform exchange $J_{r}=2 /(4 n-1)$ in Eq. 3 between all spins in different sublattices, as in the Lieb-Mattis model, ${ }^{43}$ and exchange $-J_{r}$ between all spins in the same sublattice. Uniform normalized exchange is the $\alpha=0$ limit of Eq. 3 , which can readily be written as

$$
H(4 n)=\frac{S^{2}-2 S_{\mathrm{A}}^{2}-2 S_{\mathrm{B}}^{2}+3 n}{(4 n-1)}
$$

The integer ranges are $0 \leq S \leq 2 n, 0 \leq S_{\mathrm{A}}, S_{\mathrm{B}} \leq n$ and the index $\alpha$ has been omitted. The GS is evidently always in the $S=0$ sector. In the absence of frustration, the GS is a linear combination of $2 n+1$ states with $S_{\mathrm{A}}=$ $S_{\mathrm{B}}=n$ and $z$ components $M_{\mathrm{B}}=-M_{\mathrm{A}}$. The singlet GS has antiferromagnetic coupling between sublattices with perfect ferromagnetic order.

The $g$ terms in Eq. 1 correspond to two linear HAFs with $\mathrm{PBC}$ and antiferromagnetic exchange $\mathrm{g}$ between neighbors in sublattices of $2 n$ spins. The eigenstates of the frustrated spin chain $H(4 n)+g H_{2}$ are product functions $\left|r, S_{\mathrm{A}}, 2 n\right\rangle\left|r^{\prime}, S_{\mathrm{B}}, 2 n\right\rangle$ of HAF states $r$, $r^{\prime}=1,2 \ldots$ in sectors with spin $S_{\mathrm{A}}, S_{\mathrm{B}}$. The energy of the singlet GS in the sector with $S_{\mathrm{A}}=S_{\mathrm{B}}$ is

$$
E\left(0, S_{\mathrm{A}}, g\right)=-\frac{4 S_{\mathrm{A}}\left(S_{\mathrm{A}}+1\right)-3 n}{(4 n-1)}+2 g E_{0}\left(S_{\mathrm{A}}, 2 n\right)
$$

where $E_{0}\left(S_{\mathrm{A}}, 2 n\right)$ is the lowest total energy for $S_{\mathrm{A}} \leq n$. Increasing g leads to crossovers of $E\left(0, S_{\mathrm{A}}, 4 n\right)$ between $S_{\mathrm{A}}=n$ and $m<n$. The degeneracy between $S_{\mathrm{A}}=n$ and 1 occurs at the smallest frustration that we call $g_{1}(4 n)$. The relation $E\left(0, n, g_{1}\right)=E\left(0,1, g_{1}\right)$ gives

$$
g_{1}(4 n)=\frac{n+1}{(4 n-1)} \frac{(1-2 / n(n+1))}{\left(1 / 4-E_{0}(1,2 n) / 2 n\right)}
$$

Since the infinite HAF has $E_{\mathrm{m}}=0$ and energy per site is $-\ln 2+1 / 4$, the first order transition from $S_{\mathrm{A}}=n$ to $S_{\mathrm{A}}=1$ occurs at $g_{\mathrm{c}}=1 /(4 \ln 2)$. The next transition with increasing $\mathrm{g}$ is from $S_{\mathrm{A}}=1$ to $S_{\mathrm{A}}=0$. The relation $E\left(0,1, g_{10}\right)=E\left(0,0, g_{10}\right)$ leads to

$$
\begin{aligned}
g_{10}(4 n)= & \frac{4}{(4 n-1) E_{\mathrm{m}}(2 n)} \rightarrow \\
& \frac{4}{\pi^{2}}\left(\frac{4 n}{4 n-1}\right) \frac{1}{(1-1 / 2 \ln (2 n))}
\end{aligned}
$$

where $E_{\mathrm{m}}(2 n)$ is the singlet-triplet gap. The size dependence is more delicate. The Bethe ansatz for finite systems gives $^{23} E_{\mathrm{m}}(2 n)$ to leading order and the second expression $^{22}$ in Eq. A.4. All spin correlations in Eq. 2 vanish rigorously in the infinite chains for $g>g_{10}=$ $4 / \pi^{2}$, when both sublattices are in the singlet GS.

The lowest triplet excitation has $S=1$ in Eq. A.2 and constant $E_{\mathrm{m}}=2 /(4 n-1)$ for $g<g_{10}(4 n)$. Since the sublattice spin is finite, the triplet does not require changing $\mathrm{S}_{\mathrm{A}}$ or $\mathrm{S}_{\mathrm{B}}$. The sublattices are in the singlet GS for $g>g_{10}(4 n)$, however, and $E_{\mathrm{m}}(g, 4 n)$ is given by Eq. 5 in the text. The second term is the $H(4 n)$ contribution. The triplet is doubly degenerate, localized on either sublattice, with $S=1$ and $S_{\mathrm{A}}+S_{\mathrm{B}}=1$. For $g>g_{1}(4 n)$, the singlet linear combination of a triplet on each sublattice leads to the excitation energy $E_{\sigma}(\mathrm{g}, 4 n)$,

$$
\begin{array}{r}
E_{\sigma}(g, 4 n)=|E(0,1, g)-E(0,0, g)|= \\
\left|2 g E_{\mathrm{m}}(2 n)-8 /(4 n-1)\right|
\end{array}
$$

These excitations of the analytical model are related in Fig. 3 to the $\alpha=1$ model that does not conserve sublattice spin. 


\section{REFERENCES}

1. (a) M. Nakamura, Phys. Rev. B 61 (2000) 16377-16392; (b) M. Nakamura, J. Phys. Soc. Jpn. 68 (1999) 3123-3126.

2. P. Sengupta, A. W. Sandvik, and D. K. Campbell, Phys. Rev. B. 65 (2002) 155113.

3. Y. Z. Zhang, Phys. Rev. Let. 92 (2004) 246404

4. S. Glocke, A. Klumper, and J. Sirker, Phys. Rev. B 76 (2007) 155121.

5. (a) M. Kumar, S. Ramasesha, and Z. G. Soos, Phys. Rev. B. 79 (2009) 035102; (b) M. Kumar and Z. G. Soos, Phys. Rev. B 82 (2010) 155144.

6. A. W. Sandvik, Phys. Rev. Lett. 104 (2010) 137204.

7. K. Okamoto and K. Nomura, Phys. Lett. A 169 (1992) 433-437.

8. C. K. Majumdar and D. K. Ghosh, J. Math. Phys. 10 (1969) 1399-1402.

9. Z. G. Soos and S. Mazumdar, Phys. Rev. B 18 (1978) 19912003.

10. J. B. Torrance, A. Girlando, J. J. Mayerle, J. I. Crowley, V. Y. Lee, P. Batail, and S. J. LaPlaca, Phys. Rev. Lett. 47 (1981) 1747-1750.

11. (a) Z. G. Soos, Ann. Rev. Phys. Chem. 25 (1974) 121-153; (b) A. Girlando and A. Painelli, Phys. Rev. B 34 (1986) 2131-2139; (c) N. Nagaosa, J. Phys. Soc. Jpn. 55 (1986) 27542764, ibid. 55 (1986) 3488.

12. (a) A. Girlando, A. Painelli, S. A. Bewick, and Z. G. Soos, Synth. Met. 141 (2004) 129-138, and references therein; (b) S. Horiuchi, R. Kumai, Y. Okimoto, and Y. Tokura, Chem. Phys. 325 (2006) 78-91, and references therein.

13. Z. G. Soos and A. Painelli, Phys. Rev. B 75 (2007) 155199, and references therein.

14. D. J. Klein and W. A. Seitz, Phys. Rev. B 8 (1973) 2236-2247.

15. P. G. J. van Dongen, Phys. Rev. B 49 (1994) 7904-7915.

16. (a) J. E. Hirsch, Phys. Rev. Lett. 53 (1984) 2327-2330; (b) J. E. Hirsch, Phys. Rev. B 31 (1985) 6022-6031.

17. (a) H. Bethe, Z. Phys. 71 (1931) 205-226; (b) L. Hulthen, Ark. Mat., Astron. Fys. A 26, (1938) 11.

18. M. Takahashi, Thermodynamics of One-Dimensional Solvable Models, (Cambridge University Press, Cambridge, 1999).

19. D. C. Johnston, R. K. Kremer, M. Troyer, X. Wang, A. Klü mper S. L. Bud'ko, A. F. Panchula, and P. C. Canfield, Phys. Rev. B 61 (2000) 9558-9606.

20. (a) B. S. Shastry and B. Sutherland, Phys. Rev. Lett. 47(1981) 964-967; (b) I. Affleck, T. Kennedy, E. H. Lieb, and H. Tasaki, Commun. Math. Phys. 115 (1988) 477-528.

21. M. Kumar, S. Ramasesha, and Z. G. Soos, Phys. Rev. B 81 (2010) 054413.

22. M. Kumar and Z. G. Soos, Phys. Rev. B 88 (2013) 134412.

23. F. Woynarovich and H-P. Eckle, J. Phys A: Math. Gen. 20 (1987) L97.

24. N. Laflorencie, I. Affleck, and M. Berciu, J. Stat. Mech. (2005) P12001.

25. (a) F. D. M. Haldane, Phys. Rev. Lett. 60 (1988) 635-638; (b) B. S. Shastry, Phys. Rev. Lett. 60 (1988) 639-642.

26. (a) A. D. McLachlan, Mol. Phys. 2 (1959) 276; (b) O. J. Heilmann and E. H. Lieb, Trans. N.Y. Acad. Sci. 33 (1971) 116-149; (c) S. R. Bondeson and Z. G. Soos, J. Chem. Phys. 71 (1979) 380

27. K. M. Tam, S. W. Tsai, and D. K. Campbell, Phys. Rev. Lett. 96 (2006) 036408.

28. Y. Anusooya-Pati, Z. G. Soos, and A. Painelli, Phys. Rev. B 63 (2001) 205118.

29. Z. G. Soos, S. Kuwajima, and R. H. Harding, J. Chem. Phys. 85 (1986) 601-610.

30. M. Fabrizio, A. O. Gogolin, and A. A. Nersesyan, Phys. Rev. Lett. 83 (1999) 2014-2017.

31. S. R. Manmana, V. Meden, R. M. Noack, and K. Schonhammer, Phys. Rev. B. 70 (2004) 155115.

32. A. Painelli and Z.G. Soos, Chem. Phys. 325 (2006) 48-59.

33. A. J. Heeger, S. Kivelson, J. R. Schrieffer, and W. P. Su, Rev. Mod. Phys. 60 (1988) 781-850, and references therein.

34. Z. G. Soos, D. Mukhopadhyay, A. Painelli, and A. Girlando, in Handbook of Conducting Polymers, $2^{\text {nd }}$ Ed., edited by T. Skotheim, R. Elsenbaumer, and J. Reynolds (Marcel Dekker, 1998), p. 165 .

35. (a) M. J. Rice, Solid State Commun. 31 (1979) 93-98; (b) A. Painelli and Z. G. Soos, Chem. Phys. 325 (2006) 48-59.

36. (a) J. W. Bray, L. V. Interrante, I. S. Jacobs, J. C. Bonner, in: J. S. Miller (Ed.), Extended Linear Chain Compounds, vol. 3, Plenum Press, New York, 1983, p. 353; (b) M. Hase, I. Terasaki, and K. Uchinokura, Phys. Rev. Lett. 70 (1993) 3651-3654.

37. (a) Z. G. Soos and S. A. Bewick, Chem. Phys. Lett. 421 (2006) 210-214; (b) S. A. Bewick and Z. G. Soos, Chem. Phys. 325 (2006) 60-70

38. S. A. Bewick and Z. G. Soos, J. Phys. Chem. B 110, (2005) 18748-18757.

39. M. Kumar, B. J. Topham, R. H. Yu, Q. B. Dang Ha, and Z. G. Soos, J. Chem. Phys. 134 (2011) 234304.

40. T. M. McQueen, D. M. Ho, C. Jimenez Cahua, R. J. Cava, R. A. Pascal, Jr., and Z. G. Soos, Chem. Phys. Lett. 475 (2009) 44- 48.

41. (a) M. Hase, H. Kuroe, K. Ozawa, O. Suzuki, H. Kitazawa, G. Kido, and T. Sekine, Phys. Rev. B 70 (2004) 104426; (b) H. T. Lu, Y. J.Wang, S. Qin, and T. Xiang, Phys. Rev. B 74 (2006) 134425; (c) S. - L. Drechsler, O. Volkova, A. N. Vasiliev, N. Tristan, J. Richter, M. Schmitt, H. Rosner, J. Málek, R. Klingeler, A. A. Zvyagin, and B. Büchner, Phys. Rev. Lett. 98 (2007) 077202; (d) S. E. Dutton, M. Kumar, M. Mourigal, Z. G. Soos, J. - J. Wen, C. L. Broholm, N. H. Andersen, Q. Huang, M. Zbiri, R. Toft-Petersen, and R. J. Cava, Phys. Rev. Lett. 108 (2012) 187206.

42. (a) J. Sirker, Phys. Rev. B 81, (2010) 014419; (b) S. W. Cheong and M. Mostovoy, Nat. Mater. 6 (2007) 13; S. Furukawa, M. Sato, and A. Furusaki, Phys. Rev. B 81 (2010) 094430; (c) M. Kumar and Z. G. Soos, Phys. Rev. B 85 (2012) 144415.

43. E. Lieb and D. Mattis, J. Math. Phys. 3 (1962) 749-751. 